\title{
Simplified Method for the Screening of Technological Maturity of Red Grape and Total Phenolic Compounds of Red Grape Skin: Application of the Characteristic Vector Method to Near-Infrared Spectra
}

\author{
Julio Nogales-Bueno, ${ }^{\dagger}$ Fernando Ayala, $^{\dagger}$ José Miguel Hernández-Hierro, $^{\dagger}$ \\ Francisco José Rodríguez-Pulido, ${ }^{\dagger}$ José Federico Echávarri, ${ }^{\ddagger}$ and Francisco José Heredia*, ${ }^{\dagger}$ \\ ${ }^{\dagger}$ Food Colour and Quality Laboratory, Department of Nutrition and Food Science, Facultad de Farmacia, Universidad de Sevilla, \\ 41012 Sevilla, Spain \\ ${ }^{\ddagger}$ Laboratory of Colour La Rioja, Universidad de La Rioja, 26006 Logroño, Spain
}

\begin{abstract}
Characteristic vector analysis has been applied to near-infrared spectra to extract the main spectral information from hyperspectral images. For this purpose, 3, 6, 9, and 12 characteristic vectors have been used to reconstruct the spectra, and root-mean-square errors (RMSEs) have been calculated to measure the differences between characteristic vector reconstructed spectra (CVRS) and hyperspectral imaging spectra (HIS). RMSE values obtained were 0.0049, 0.0018, 0.0012 , and 0.0012 $[\log (1 / R)$ units $]$ for spectra allocated into the validation set, for $3,6,9$, and 12 characteristic vectors, respectively. After that, calibration models have been developed and validated using the different groups of CVRS to predict skin total phenolic concentration, sugar concentration, titratable acidity, and $\mathrm{pH}$ by modified partial least-squares (MPLS) regression. The obtained results have been compared to those previously obtained from HIS. The models developed from the CVRS reconstructed from 12 characteristic vectors present similar values of coefficients of determination (RSQ) and standard errors of prediction (SEP) than the models developed from HIS. RSQ and SEP were 0.84 and $1.13 \mathrm{mg} \mathrm{g}^{-1}$ of skin grape (expressed as gallic acid equivalents), 0.93 and $2.26{ }^{\circ}$ Brix, 0.97 and $3.87 \mathrm{~g} \mathrm{~L}^{-1}$ (expressed as tartaric acid equivalents), and 0.91 and 0.14 for skin total phenolic concentration, sugar concentration, titratable acidity, and $\mathrm{pH}$, respectively, for the models developed from the CVRS reconstructed from 12 characteristic vectors.
\end{abstract}

KEYWORDS: near-infrared spectroscopy, hyperspectral imaging, grape maturity, characteristic vector analysis, chemometrics

\section{INTRODUCTION}

Hyperspectral imaging is currently a proven analytical tool for food quality and safety control. There are many studies that use hyperspectral imaging to control, detect, or measure parameters of interest in food. ${ }^{1-3}$ Some studies even describe how this technology can be applied to real online industrial processes., ${ }^{4,5}$ However, some of the main drawbacks of the aforesaid technology are the huge amount of generated data, which implies slow data processing, and the high cost of hyperspectral imaging systems. Thus, a number of the current food studies have been geared toward identification of important wavelengths for the development of low-cost multi-spectral imaging systems. $^{3,6,7}$

Multi-spectral imaging reduces the total volume of data, achieving fast image acquisition and simple algorithms for image processing and decision-making processes. In practice, this means acquiring images with relatively low spatial resolutions at a few important wavelengths. ${ }^{1}$ There are several alternatives to identify these important wavelengths from hyperspectral imaging. The main methodologies applied to select wavelengths in hyperspectral imaging are partial leastsquares regression (PLSR), stepwise regression (SWR), successive projections algorithm (SPA), uninformative variable elimination (UVE), second derivative spectra (2ndD), and band ratio (BR). ${ }^{7}$

Rajkumar et al. ${ }^{8}$ used the PLSR method to select eight wavelengths and predict some quality parameters in banana fruit obtaining correlation coefficients (RSQ) higher than 0.85 . Sugiyama et al. ${ }^{9}$ differentiated foreign substances from blueberries using a SWR methodology to identify only two important infrared wavelengths. Fang et al. ${ }^{10}$ compared different SPA methodologies to extract the most important wavelengths and detect tomato growth state and diseases. They obtained a RSQ coefficient of 0.94 using only eight wavelengths. In addition, the UVE algorithm has been applied to predict apple firmness in wavelength selection in hyperspectral imaging. Models developed from the selected wavelengths achieve better results than models developed from all of the wavelengths (RSQ of 0.81 versus 0.79). ${ }^{11}$ Finally, simpler methodologies, such as $2 \mathrm{ndD}$ and $\mathrm{BR}$, has been applied to select the most important wavelengths in red meat and citrus spectra, respectively. ${ }^{12,13}$ 
However, other alternatives can be used to reduce the volume of data that hyperspectral imaging acquires. Characteristic vector analysis has been used to identify the most important wavelengths in the visible region by several authors. Then, these authors could reconstruct the visible spectra of other samples from these important wavelengths. In the visible region, characteristic vector analysis has been applied to the reflectance spectra of wines, brandies, vinegars, liquid honeys, and orange juices. ${ }^{14-17}$ The aforesaid reflectance spectra were acquired by means of different spectroscopic tools (i.e., spectrophotometry and spectroradiometry). To check the accuracy of the reconstructed spectra, CIELAB coordinates were calculated from experimental and reconstructed spectra. Then, color differences were also calculated using the coordinates obtained from the experimental spectra as reference coordinates and the coordinates obtained after applying the characteristic vector method as recalculated coordinates.

However, to the best of our knowledge, characteristic vector analysis has not been applied to near-infrared spectra to identify the most important wavelengths and reconstruct the spectra. Nonetheless, characteristic vector analysis is an interesting tool to be applied to near-infrared spectra. By means of this tool, important wavelengths could be found and low-cost multispectral imaging systems could be developed. Thus, it would be possible to measure parameters of interest in food, reducing the total volume of data, using this method in a number of food fields, such as grape and wine industry.

Grape maturity is one of the most fundamental aspects that have influence on the future of wine quality. It is composed of technological and phenolic maturity, especially phenolic maturity of grape skin. Technological maturity is mainly related with the sugar concentration, titratable acidity, and $\mathrm{pH}$. The sugar concentration determines the potential alcohol strength. The titratable acidity and $\mathrm{pH}$ help to control the wine quality and color. Phenolic maturity shows the ripening degree for the skins, pulp, and seeds, taking into account the phenolic composition. $^{18,19}$

Nowadays, at wineries, maturity of grapes is usually controlled using classic physical and chemical analyses (e.g., refractometric, volumetric, chromatographic, or spectrophotometric analyses). As preliminary approaches, we have described some methods to screen grape maturity by means of nearinfrared hyperspectral imaging. ${ }^{20-22}$ These are useful and nondestructive hyperspectral methods. However, as described above, hyperspectral imaging involves high costs and achieves a high volume of data, while multi-spectral imaging reduces these problems considerably.

The main aim of this study is to check the feasibility of using characteristic vector analysis in the near-infrared spectral region. This method may provide the most important wavelengths to reconstruct the whole spectrum from discrete measurement. Furthermore, the spectra obtained using this simplified method are evaluated to screen technological maturity of red grape and total phenolic contents of red grape skin. To our knowledge, this is the first time that characteristic vector analysis has been applied to near-infrared spectra to face the aforementioned goals.

\section{MATERIALS AND METHODS}

Samples. Vitis vinifera L. cv. Tempranillo is the most often grown red grape cultivar in Spain for producing quality red wines, and $V$. vinifera L. cv. Syrah is a resistant cultivar to warm climatic conditions. ${ }^{23,24}$ Tempranillo and Syrah samples were collected at different dates from mid-July to early September in the 2012 vintage. In this way, samples were collected at different stages of physiological maturity and from two vineyards located in the Condado de Huelva Designation of Origin D.O. (Andalusia, Spain) as previously described elsewhere by Nogales-Bueno et al. ${ }^{21}$ Briefly, 16 and 17 dates were taken into account for Tempranillo and Syrah, respectively, and three groups of at least 150 berries were collected from several bunches for each date and vineyard. With the aim of achieving representative samples, the berries were collected from both sides (sunlight and shade) of vines located in different rows within the vineyard. Edge rows and the first two vines in a row were avoided. Berries were collected from the top, middle, and bottom of the cluster. A total of 99 samples were collected, $3 \times 16$ Tempranillo samples and $3 \times 17$ Syrah samples. From the three samples of each date, one (33\%) was randomly allocated into the validation set and the other two (66\%) were allocated to the calibration set.

Near-Infrared Hyperspectral Imaging. The hyperspectral imaging device (Infaimon S.L., Barcelona, Spain) comprised a Xenics XEVA-USB InGaAs camera $(320 \times 256$ pixels, Xenics Infrared Solutions, Inc., Leuven, Belgium) and a spectrograph (Specim ImSpector N17E Enhanced, Spectral Imaging, Ltd., Oulu, Finland) covering the spectral range between 900 and $1700 \mathrm{~nm}$ (spectral resolution of $3.25 \mathrm{~nm}$ ). The hyperspectral image of each sample was recorded. The equipment and procedure used for image recording are described in detail elsewhere by Hernández-Hierro et al. ${ }^{20}$

After the calibration and segmentation process, the average spectral profile for each sample was saved. Noisy wavebands at both extremes of the spectral range were removed. Thus, only spectral data in the resulting effective wavelength of 950-1650 $\mathrm{nm}$ regions were used in data analysis because of reduced efficiency outside this range in the used device. Finally, the average spectra were transformed into $\log (1 /$ $R$ ) units, and 99 near-infrared absorbance spectra were obtained, with each of them composed of 215 wavelengths.

Reference Parameters. Reference parameters were total phenolic concentration in grape skins, sugar concentration, titratable acidity, and $\mathrm{pH}$. The total phenolic concentration in grape skins was determined using the Folin-Ciocalteu method, ${ }^{25}$ and it was expressed as gallic acid equivalents per gram of grape skin. This method was applied to an aqueous extract obtained from grape skin. Grape skins were separated manually from the whole grapes. Afterward, $1 \mathrm{~g}$ of grape skin was macerated in $10 \mathrm{~mL}$ of methanol containing $0.1 \% 12 \mathrm{M}$ $\mathrm{HCl}$. Methanolic phases were centrifuged $(3000 \mathrm{rpm}$ for $10 \mathrm{~min}$ ) and successively pooled; approximately $2 \mathrm{~mL}$ of water was added; and the extract was concentrated under vacuum at $30{ }^{\circ} \mathrm{C}$ until methanol was removed and finally made up to $10 \mathrm{~mL}$ with ultrapure water. Technological maturity parameters were determined using the analytical methods recommended by the International Organisation of Vine and Wine (OIV) ${ }^{26}$ as described elsewhere by Nogales-Bueno et al. ${ }^{21}$

Chemometrics Tools: Characteristic Vector Method. A mathematical statement of the characteristic vector analysis ${ }^{27}$ might be given as follows:

Hyperspectral imaging spectra (HIS) are near-infrared absorbance spectra obtained from the hyperspectral device. An individual response data $\rho_{\lambda}$ (absorbance spectrum between 950 and $1650 \mathrm{~nm}$, at $\Delta \lambda=3.25$ $\mathrm{nm}$ ) is available for $r$ (215) levels of the variable $\lambda$ (wavelength) and can be plotted as a response curve. Therefore, for each sample, the $r$ values of $\rho_{\lambda}$ constitute a one-row $r$-column vector of response data. For $n$ samples, the response vectors can be arrayed to form a data matrix of $n$ rows and $r$ columns.

If the variance-covariance matrix $\mathbf{S}$ from the original $(n \times r)$ data matrix is computed, characteristic vectors (or eigenvectors) are the solutions of the equation

$$
\operatorname{det}(\mathbf{S}-\mathbf{L I})=0
$$

where $\mathbf{L}$ is the diagonal matrix $(r \times r)$ of the characteristic root and $\mathbf{I}$ is the $(r \times r)$ unit matrix.

It is possible to find a set of $p$ characteristic vectors, $(p<r)$, which, added in the proper amounts, will adequately approximate any of the 
original family of response vectors. The result is that the components of the generic vectors (i.e., the absorbance or reflectance spectra) can be expressed as

$$
\rho_{\lambda}=M_{1} V_{1 \lambda}+M_{2} V_{2 \lambda}+\ldots+M_{p} V_{p \lambda}, \quad p<r
$$

where $V_{i \lambda}$ represents the components of the characteristic $i$ th vector and $M_{i}$ represents the specific coefficients, called scalar multiples, of each reconstructed vector. The $M_{i}$ coefficients depend upon the absorbance values at as many wavelengths as characteristic vectors have been needed. These wavelengths are selected to maximize the accuracy of the characteristic vector reconstructed spectra (CVRS).

This analysis must be applied to a large number $(n)$ of experimental curves. The iterative mathematical procedure calculates the characteristic vectors, the $M_{i}$ coefficients to reconstruct each of these curves, and the percentage of variability among the family of homologous response curves explained for each characteristic vector.

The derivation of the vectors ensures that the first vector accounts for the largest amount of total response variability, the second vector accounts for the second-largest amount of variability, etc.

The inconvenience of this methodology is that it allows us to reconstruct the spectra of samples only from the calibration set (also called model group). If the results are to be applied to spectra of samples other than those used previously, it is necessary to calculate the corresponding $M_{i}$ coefficients in a different way.

To solve this issue, characteristic vectors are calculated. Then, expressions to calculate the $M_{i}$ coefficients are obtained. These expressions are functions of the absorbance or reflectance values at as many wavelengths as characteristic vectors have been needed. Therefore, these expressions allow us to calculate the $M_{i}$ coefficients for the samples from the calibration set as well as for other samples from a different set called validation set. In this way, one of the requirements from the statistical methodology is completed that requires testing of a model with samples different from those used to obtain the model. Therefore, when the characteristic vector method is established, the absorbance or reflectance values at only a few wavelengths are necessary to reconstruct a spectrum.

Finally, to check the accuracy of the CVRS, root-mean-square error (RMSE) is calculated. This error measures the differences between CVRS and HIS for the calibration and validation sets. However, a small RMSE does not provide sufficient guarantee to conclude that the structural information offered by the near-infrared HIS may be kept.

Chemometrics Tools: Modified Partial Least-Squares (MPLS) Method. To check that CVRS keep the main structural information related to molecular bonds after the application of the characteristic vector method, a checking process has been purposed. Using the calibration models described in our previous study, ${ }^{21}$ it was checked how, from CVRS, the aforementioned reference parameters were predicted. Then, the differences between the reference parameters predicted from the former data (HIS data) and the reference parameters predicted from the CVRS were checked.

Briefly, in our previous study, ${ }^{21}$ it was established what spectral pretreatment was the best for each reference parameter. For red grapes, standard normal variate (SNV), detrend, or spectral derivative pretreatments were used to predict the different reference parameters. These pretreatments were shown to be the most effective tools to remove scattering effects. Then, calibration models were performed using MPLS regression, and the aforementioned reference parameters were predicted.

Consequently, these spectral pretreatments have been applied to the CVRS; quantitative calibrations have been developed by MPLS regression; and external validations have been carried out. Then, RSQ coefficients and SEP errors have been calculated and compared to the statistical parameters obtained from HIS.

To study the feasibility of the characteristic vector method to be used in the near-infrared region, this method has been developed from $3,6,9$, and 12 characteristic vectors and the aforesaid process has been carried out from the different sets of CVRS.

\section{RESULTS AND DISCUSSION}

Reference Parameters. The total phenolic concentration ranged from 2.2 to $14.3 \mathrm{mg} \mathrm{g}^{-1}$ of grape skin expressed as gallic acid equivalents. The sugar concentration ranged from 4.1 to $25.4{ }^{\circ}$ Brix. Titratable acidity ranged from 3.1 to $52.9 \mathrm{~g} \mathrm{~L}^{-1}$ expressed as tartaric acid equivalents. Finally, $\mathrm{pH}$ ranged from 2.5 to 3.8 .

Spectral Samples and Spectral Reconstruction Process. After hyperspectral imaging acquisition, near-infrared HIS were combined into the spectral matrix $(99 \times 215)$, this spectral matrix was split into calibration $(66 \times 215)$ and validation $(33 \times 215)$ matrixes. Then, the characteristic vector method was applied to the calibration matrix, and the first 12 characteristic vectors were calculated. Both, calibration and validation matrixes were reconstructed using the first $3,6,9$, and 12 characteristic vectors successively. The expressions and used wavelengths to calculate the $M_{i}$ coefficients are available in the Supporting Information.

Figure 1 shows the first 12 characteristic vectors calculated from the HIS allocated into the calibration set. These vectors

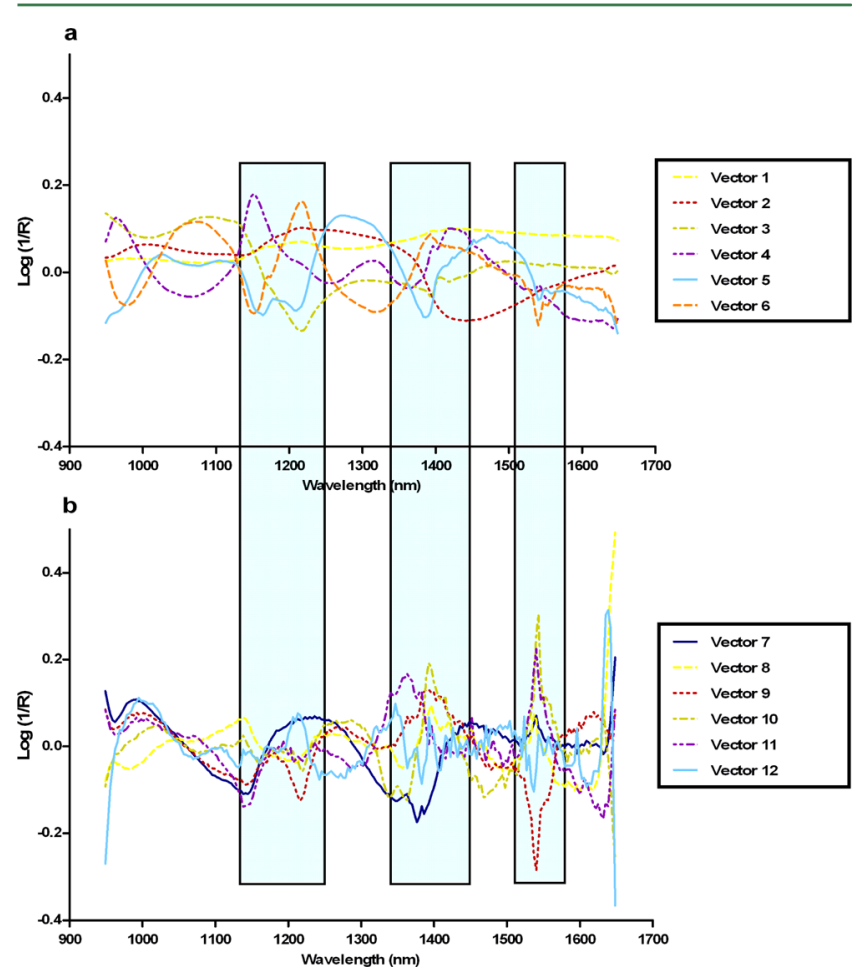

Figure 1. Characteristic vectors calculated from the spectra allocated into the calibration set. The first 6 characteristic vectors are shown in panel $a$, and the next 6 characteristic vectors are shown in panel $b$.

form the basis of a vector space, and thus, the HIS allocated into the calibration set belong to this vector space. The first 6 characteristic vectors are shown in Figure 1a, and the next 6 characteristic vectors are shown in Figure $1 \mathrm{~b}$. A strong feature of the characteristic vectors was the pattern around 1150, 1350, and $1550 \mathrm{~nm}$ wavelengths. The pattern around these wavelengths is mainly related to bands of the $-\mathrm{CH}$ and $-\mathrm{OH}$ functional groups. ${ }^{28}$ Table 1 shows the RMSE values of the reconstruction of the calibration and validation matrixes. As expected, in all cases, the reconstruction of the calibration matrix is better than the reconstruction of the validation matrix. Nevertheless, all of these errors are really low; in fact, if a HIS is 
Table 1. RMSE Obtained in the Reconstruction of the Calibration and Validation Matrixes from 3, 6, 9, and 12 Characteristic Vectors

\begin{tabular}{ccccc} 
RMSE $^{a}$ & 3 vectors & 6 vectors & 9 vectors & 12 vectors \\
$\begin{array}{cccc}\text { calibration set } \\
(66 \text { samples })\end{array}$ & 0.004778 & 0.001513 & 0.000819 & 0.000746 \\
$\begin{array}{c}\text { validation set } \\
(33 \text { samples })\end{array}$ & 0.004873 & 0.001790 & 0.001149 & 0.001223 \\
${ }^{a} \mathrm{RMSE}=$ root-mean-square error $[\log (1 / R)$ & units $].$ \\
\hline
\end{tabular}

plotted together with its respective CVRS, there are very few differences between them. However, it is not certain that CVRS keep all of the structural information related to molecular bonds. To check this, calibration models were developed from the different sets of CVRS.

Development of MPLS Calibrations. Table 2 resumes the calibration statistical descriptors for the different equations developed. Equations have been developed for each reference parameter using five different groups of spectra: the group of HIS and four groups of CVRS reconstructed from the first 3, 6, 9 , and 12 characteristic vectors. In this table, it can be observed how models developed from CVRS achieve different results depending upon the number of characteristic vectors used to reconstruct them. Groups of CVRS reconstructed from 3 and 6 characteristic vectors show high values of error in the crossvalidation process (SECV) and low correlation coefficients (RSQ). In addition, these equations have needed less PLS factors than the rest of spectral groups. This means that the spectral variability of the independent variable $(X)$ related to the dependent variable $(Y)$ is lower in groups of CVRS reconstructed from 3 and 6 vectors than in the rest of the groups of CVRS, and this spectral variability can be explained with a small number of PLS factors. In conclusion, these two reconstructions have not been able to reproduce all of the spectral variability of the HIS related to the dependent variable (Y).

The group of CVRS reconstructed from 9 and 12 characteristic vectors shows better results. In fact, the results obtained from the group reconstructed from 12 characteristic vectors are really close to the results obtained from HIS (Table 2).

For each model developed from the different calibration sets, its robustness was tested using its respective validation set (33 samples), which did not belong to the calibration set, as external validation. Using the characteristic vector analysis described above, these 33 HIS were reconstructed from 3, 6, 9, and 12 characteristic vectors and these matrixes were used in the different validation processes. As result of these external validations, standard errors of prediction (SEP) for each reference variable were obtained; these values were also included in Table 2. Figure 2 shows the SEP expressed as percentages. These errors follow the same pattern described for SECV: high errors for models developed from 3 and 6 characteristic vectors and lower errors for models developed from 9 and 12 vectors. For sugar, concentration errors obtained are not as low as expected; however, the model developed from 12 characteristic vectors predicts this parameter with sufficient precision. Furthermore, for total phenolic concentration and titratable acidity, SEP obtained in the model developed from 12 characteristic vectors is lower than SEP obtained in the model developed from HIS. This is perhaps due to the absence of noise in the CVRS. The characteristic vector method can reproduce the features of a spectrum linked to the chemical or physical properties of the sample. However, this method cannot reproduce the instrumental noise of a HIS, by its random nature. Consequently, in the CVRS, the instrumental noise cannot mask the features linked to the chemical or physical

Table 2. Calibration Statistical Descriptors for the Models Developed in the Near-Infrared Region Close to $950-1650 \mathrm{~nm}$

\begin{tabular}{|c|c|c|c|c|c|c|c|c|c|c|c|c|}
\hline parameters & spectra $^{a}$ & $\begin{array}{l}\text { spectral } \\
\text { pretreatments }\end{array}$ & $T$ outliers & PLS factors & $N^{b}$ & $\begin{array}{l}\text { estimated } \\
\text { minimum }\end{array}$ & $\mathrm{SD}^{c}$ & $\begin{array}{l}\text { estimated } \\
\text { maximum }\end{array}$ & $\mathrm{SEC}^{d}$ & $\mathrm{RSQ}^{e}$ & $\mathrm{SECV}^{f}$ & $\mathrm{SEP}^{g}$ \\
\hline \multirow{5}{*}{$\begin{array}{l}\text { total } \\
\text { phenols }\end{array}$} & 3 vectors & \multirow{5}{*}{$\begin{array}{l}\text { SNV and detrend } \\
\quad 0,0,1,1\end{array}$} & 4 & 1 & 62 & 0 & 2.95 & 16.95 & 2.00 & 0.54 & 2.07 & 1.86 \\
\hline & 6 vectors & & 2 & 2 & 64 & 0 & 3.02 & 16.81 & 1.72 & 0.68 & 1.79 & 1.56 \\
\hline & 9 vectors & & 0 & 4 & 66 & 0 & 3.13 & 17.30 & 1.29 & 0.82 & 1.42 & 1.35 \\
\hline & 12 vectors & & 1 & 4 & 65 & 0 & 3.08 & 17.06 & 1.22 & 0.84 & 1.35 & 1.13 \\
\hline & HIS $^{i}$ & & 3 & 6 & 63 & 0 & 2.99 & 16.69 & 1.01 & 0.89 & 1.07 & 1.23 \\
\hline \multirow[t]{5}{*}{${ }^{\circ}$ Brix } & 3 vectors & \multirow[t]{5}{*}{ none $2,5,5,1$} & 4 & 1 & 62 & 0.85 & 5.49 & 33.78 & 3.86 & 0.50 & 3.97 & 3.70 \\
\hline & 6 vectors & & 2 & 3 & 64 & 0 & 6.00 & 34.60 & 2.65 & 0.80 & 2.90 & 2.62 \\
\hline & 9 vectors & & 2 & 4 & 64 & 0 & 6.09 & 35.10 & 1.57 & 0.93 & 1.78 & 2.45 \\
\hline & 12 vectors & & 3 & 5 & 63 & 0 & 6.04 & 34.76 & 1.61 & 0.93 & 1.78 & 2.26 \\
\hline & HIS $^{i}$ & & 4 & 6 & 62 & 0 & 5.98 & 34.61 & 0.73 & 0.99 & 1.04 & 1.37 \\
\hline \multirow{5}{*}{$\begin{array}{l}\text { titratable } \\
\text { acidity }^{j}\end{array}$} & 3 vectors & \multirow[t]{5}{*}{ SNV $0,0,1,1$} & 8 & 3 & 58 & 0 & 10.34 & 42.50 & 6.12 & 0.65 & 6.35 & 7.08 \\
\hline & 6 vectors & & 6 & 4 & 60 & 0 & 13.69 & 54.25 & 3.24 & 0.94 & 3.33 & 4.58 \\
\hline & 9 vectors & & 6 & 8 & 60 & 0 & 12.66 & 50.32 & 1.93 & 0.98 & 2.07 & 4.24 \\
\hline & 12 vectors & & 5 & 6 & 61 & 0 & 13.49 & 53.79 & 2.38 & 0.97 & 2.62 & 3.87 \\
\hline & $\mathrm{HIS}^{i}$ & & 5 & 8 & 61 & 0 & 13.39 & 53.08 & 1.72 & 0.98 & 2.24 & 3.88 \\
\hline \multirow[t]{5}{*}{$\mathrm{pH}$} & 3 vectors & \multirow{5}{*}{$\begin{array}{l}\text { SNV and detrend } \\
1,5,5,1\end{array}$} & 6 & 2 & 60 & 2.16 & 0.33 & 4.17 & 0.19 & 0.67 & 0.20 & 0.21 \\
\hline & 6 vectors & & 3 & 6 & 63 & 2.08 & 0.35 & 4.19 & 0.16 & 0.78 & 0.17 & 0.17 \\
\hline & 9 vectors & & 0 & 6 & 66 & 2.08 & 0.36 & 4.23 & 0.13 & 0.87 & 0.14 & 0.15 \\
\hline & 12 vectors & & 3 & 8 & 63 & 2.06 & 0.36 & 4.23 & 0.11 & 0.91 & 0.12 & 0.14 \\
\hline & $\mathrm{HIS}^{i}$ & & 0 & 8 & 66 & 2.08 & 0.36 & 4.23 & 0.09 & 0.94 & 0.13 & 0.12 \\
\hline
\end{tabular}

${ }^{a}$ Spectra $=$ group of spectra used for develop the model. ${ }^{b} \mathrm{~N}=$ number of samples (calibration set). ${ }^{c} \mathrm{SD}=$ standard deviation. ${ }^{d} \mathrm{SEC}=$ standard error of calibration. ${ }^{e} \mathrm{RSQ}=$ coefficient of determination. ${ }^{f_{\mathrm{SECV}}}=$ standard error of cross-validation. ${ }^{g} \mathrm{SEP}=$ standard error of prediction (external validation). ${ }^{h}$ Total phenols $=\mathrm{mg} \mathrm{g}^{-1}$ of skin grape (expressed as gallic acid equivalents). ${ }^{i} \mathrm{HIS}=$ hyperspectral imaging spectra. ${ }^{j}$ Titratable acidity $=\mathrm{g}$ $\mathrm{L}^{-1}$ (expressed as tartaric acid equivalents). 


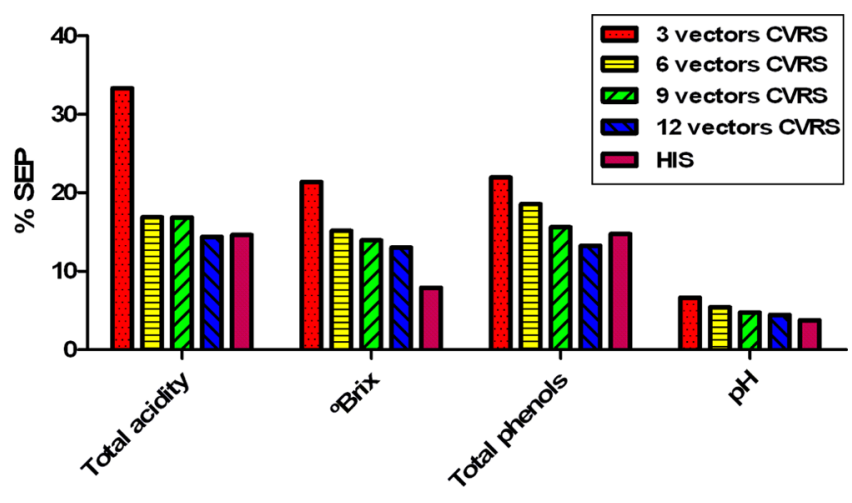

Figure 2. SEP for each reference parameter expressed as percentages, showing a comparison between the different calibration models developed.

properties of the sample, and the models developed from the CVRS reconstructed from 12 characteristic vectors can be more accurate than models developed from HIS.

To analyze the significance of differences between errors of prediction for the models developed from HIS and from the group of CVRS reconstructed from 12 characteristic vectors, a Student's $t$ test for paired values was carried out. In each case, the level of significance was obtained. When this value was greater than 0.05 (chosen as the minimum level of significance), the null hypothesis was accepted (i.e., there were no differences among the different results). The levels of significance were 0.22 for total phenolic concentration, 0.76 for sugar concentration, 0.18 for titratable acidity, and 0.13 for $\mathrm{pH}$. For all parameters, the level of significance was higher than 0.05. It may be concluded that the models developed from the group of CVRS reconstructed from 12 characteristic vectors provide significantly similar results to models developed from HIS.
Furthermore, the error of prediction (EP) of each sample was calculated to test the robustness of the developed models. For each developed model, differences between individual predicted and experimental reference parameters have been calculated and expressed as percentages. These percentages are plotted in Figure 3, which shows the distributions in error of predictions of samples allocated into the validation set. For all reference parameters, the number of samples with an error of prediction lower than $20 \%$ is very similar for the models developed from HIS and from the group of CVRS reconstructed from 12 characteristic vectors. This means that characteristic vector analysis reproduces, with only $6 \%$ of the spectral information that the hyperspectral device provides, the results reported in our previous study ${ }^{21}$ in a very satisfactory way (RSQ between 0.84 and 0.97 units and SEP between 4 and $14 \%)$. In addition, Figure 3 also shows that $\mathrm{pH}$ could be predicted with an EP of lower than $20 \%$, even using the model developed from the group of CVRS reconstructed from 3 characteristic vectors (Figure $3 \mathrm{~d}$ ).

In conclusion, simplified methods for the screening of technological maturity of red grape and total phenolic compounds of red grape skin have been described. These methods use characteristic vector analysis to reduce the volume of data that hyperspectral imaging acquires. This data reduction can, from a theoretical point of view, help to develop cheap spectral devices, such as multi-spectral systems.

The characteristic vector method allows us to reconstruct near-infrared spectra from a few characteristic vectors and wavelengths. To achieve good results, the correct number of characteristic vectors must be taken into account. The results reported here indicate that 12 characteristic vectors are sufficient to reconstruct near-infrared spectra and to develop from these spectra useful calibration models. Hence, this study is an important step to reduce the spectral information necessary for food evaluation and the cost of this technology.
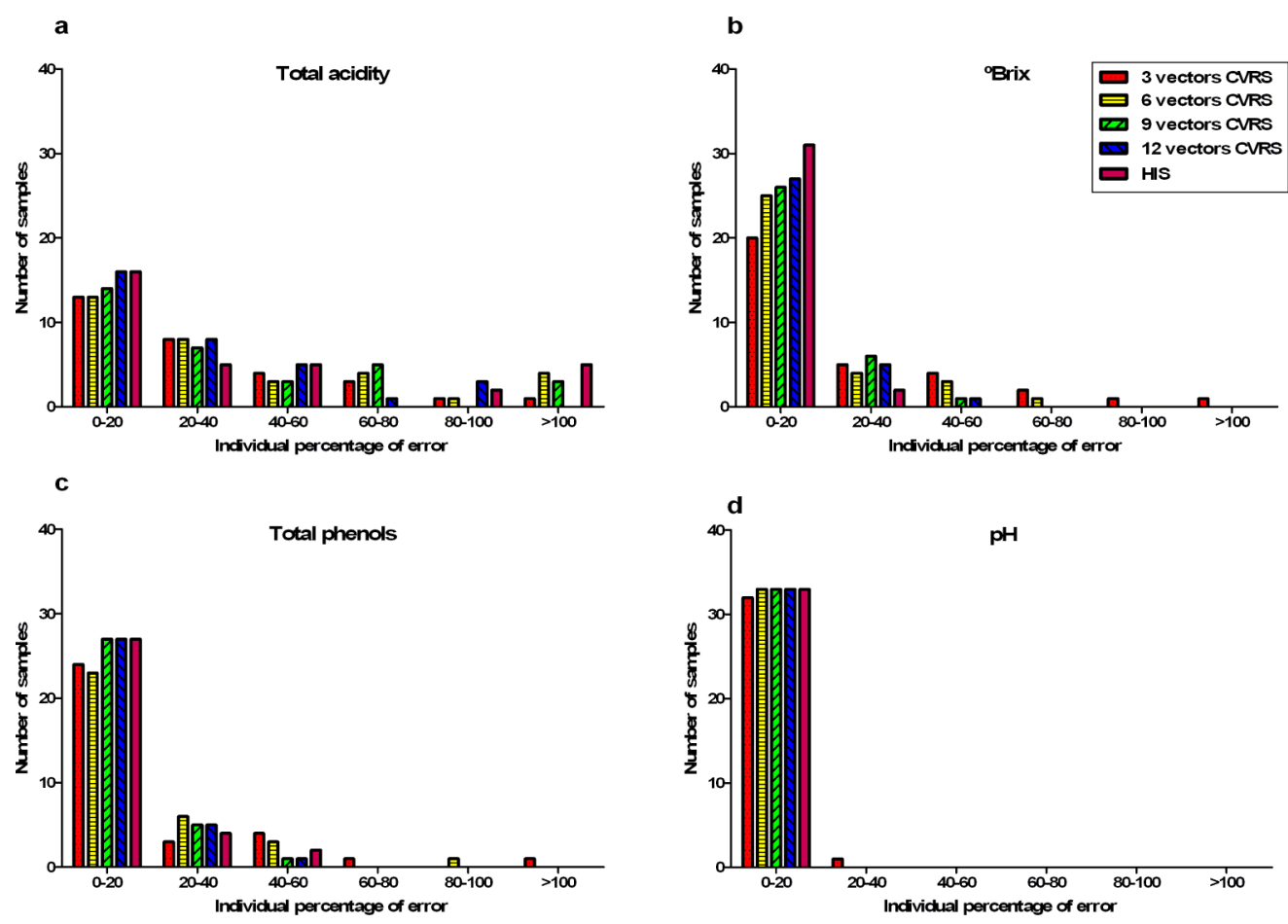

Figure 3. Frequency histograms of (a) total acidity, (b) sugar concentration, (c) total phenols, and (d) $\mathrm{pH}$ are represented. 


\section{ASSOCIATED CONTENT}

\section{S Supporting Information}

Expressions and used wavelengths to calculate the $M_{i}$ coefficients (Appendix A: Spectral Reconstruction). The Supporting Information is available free of charge on the ACS Publications website at DOI: $10.1021 /$ jf505870s.

\section{AUTHOR INFORMATION}

\section{Corresponding Author}

*Telephone: +34-9545-56495. Fax: +34-9545-56110. E-mail: heredia@us.es.

\section{Funding}

The Spanish Ministry of Economy and Competitiveness (MINECO) is thanked for the Formación de Personal Investigador (FPI) Grant (BES-2012-060192) to Julio Nogales-Bueno and Project AGL2014-58486-C2. José Miguel Hernández-Hierro thanks Universidad de Sevilla for the extension of the Juan de la Cierva postdoctoral contract. Francisco José Rodriguez-Pulido also thanks the Department of Financial Economics and Operations Management (VPPI-US), Universidad de Sevilla, for a postdoctoral grant. Junta de Andalucía is also thanked for financial support (Project P10AGR6331).

\section{Notes}

The authors declare no competing financial interest.

\section{ACKNOWLEDGMENTS}

The authors thank the technical staff of Biology Service [Servicios Generales de Investigación (SGI), Universidad de Sevilla] for the technical assistance.

\section{ABBREVIATIONS USED}

2ndD, second derivative spectra; BR, band ratio; CVRS, characteristic vector reconstructed spectra; EP, error of prediction; HIS, hyperspectral imaging spectra; MPLS, modified partial least-squares; NIRS, near-infrared spectroscopy; PLSR, partial least-squares regression; RMSE, root-meansquare error; RSQ coefficient of determination; SEC, standard error of calibration; SECV, standard error of cross-validation; SEP, standard error of prediction; SNV, standard normal variate; SPA, successive projections algorithm; SWR, stepwise regression; UVE, uninformative variable elimination

\section{REFERENCES}

(1) Qin, J.; Chao, K.; Kim, M. S.; Lu, R.; Burks, T. F. Hyperspectral and multispectral imaging for evaluating food safety and quality. $J$. Food Eng. 2013, 118, 157-171.

(2) Wu, D.; Sun, D. W. Advanced applications of hyperspectral imaging technology for food quality and safety analysis and assessment: A review-Part II: Applications. Innovative Food Sci. Emerging Technol. 2013, 19, 15-28.

(3) Gowen, A. A.; O’Donnell, C. P.; Cullen, P. J.; Downey, G.; Frias, J. M. Hyperspectral imaging-An emerging process analytical tool for food quality and safety control. Trends Food Sci. Technol. 2007, 18, 590-598.

(4) Garcia-Allende, P. B.; Conde, O. M.; Mirapeix, J.; Cobo, A.; Lopez-Higuera, J. M. Hyperspectral imaging for diagnosis and quality control in agri-food and industrial sectors. Opt. Sens. Detect. 2010, 7726, No. 77260E.

(5) Gou, P.; Santos-Garcés, E.; Høy, M.; Wold, J. P.; Liland, K. H.; Fulladosa, E. Feasibility of NIR interactance hyperspectral imaging for on-line measurement of crude composition in vacuum packed drycured ham slices. Meat Sci. 2013, 95, 250-255.
(6) Esquerre, C.; Gowen, A.; Downey, G.; O’Donnell, C. P. Wavelength selection for development of a near infrared imaging system for early detection of bruise damage in mushrooms (Agaricus bisporus). J. Near Infrared Spectrosc. 2012, 20, 537-546.

(7) Liu, D.; Sun, D. W.; Zeng, X. A. Recent advances in wavelength selection techniques for hyperspectral image processing in the food industry. Food Bioprocess Technol. 2014, 7, 307-323.

(8) Rajkumar, P.; Wang, N.; Eimasry, G.; Raghavan, G. S. V.; Gariepy, Y. Studies on banana fruit quality and maturity stages using hyperspectral imaging. J. Food Eng. 2012, 108, 194-200.

(9) Sugiyama, T.; Sugiyama, J.; Tsuta, M.; Fujita, K.; Shibata, M.; Kokawa, M.; Araki, T.; Nabetani, H.; Sagara, Y. NIR spectral imaging with discriminant analysis for detecting foreign materials among blueberries. J. Food Eng. 2010, 101, 244-252.

(10) Fang, H. Detection of activity of POD in tomato leaves based on hyperspectral imaging technology. Spectrosc. Spect. Anal. 2012, 32, 2228-2233.

(11) Wang, S.; Huang, M.; Zhu, Q. Model fusion for prediction of apple firmness using hyperspectral scattering image. Comput. Electron. Agr. 2012, 80, 1-7.

(12) Kamruzzaman, M.; Barbin, D.; Elmasry, G.; Sun, D. W.; Allen, P. Potential of hyperspectral imaging and pattern recognition for categorization and authentication of red meat. Innovative Food Sci. Emerging Technol. 2012, 16, 316-325.

(13) Li, J.; Rao, X.; Guo, J.; Ying, Y. Hyperspectral reflectance imaging for detecting citrus canker based on dual-band ratio image classification method. Proceedings of the 5th International Symposium on Advanced Optical Manufacturing and Testing Technologies: Optical Test and Measurement Technology and Equipment; Dalian, China, April 2629, 2010; pp 76562C1-76562C10.

(14) Ayala, F.; Echávarri, J. F.; Negueruela, A. I. A new simplified method for measuring the color of wines. III. All wines and brandies. Am. J. Enol. Vitic. 1999, 50, 359-363.

(15) González-Miret, M. L.; Ayala, F.; Terrab, A.; Echávarri, J. F.; Negueruela, A. I.; Heredia, F. J. Simplified method for calculating colour of honey by application of the characteristic vector method. Food Res. Int. 2007, 40, 1080-1086.

(16) Meléndez-Martinez, A. J.; Ayala, F.; Echávarri, J. F.; Negueruela, A. I.; Escudero-Gilete, M. L.; González-Miret, M. L.; Vicario, I. M.; Heredia, F. J. A novel and enhanced approach for the assessment of the total carotenoid content of foods based on multipoint spectroscopic measurements. Food Chem. 2011, 126, 1862-1869.

(17) García-Parrilla, M. C.; Ayala, F.; Echávarri, J. F.; Troncoso, A. M.; Negueruela, A. I.; Heredia, F. J. Measurement of wine vinegars' color: Application of the characteristic vector method. J. Agric. Food Chem. 1998, 46, 4238-4241.

(18) Ferrer-Gallego, R.; Hernández-Hierro, J. M.; Rivas-Gonzalo, J. C.; Escribano-Bailón, M. T. Influence of climatic conditions on the phenolic composition of Vitis vinifera L. cv. Graciano. Anal. Chim. Acta 2012, 732, 73-77.

(19) Meléndez, E.; Ortiz, M. C.; Sarabia, L. A.; Íñiguez, M.; Puras, P. Modelling phenolic and technological maturities of grapes by means of the multivariate relation between organoleptic and physicochemical properties. Anal. Chim. Acta 2013, 761, 53-61.

(20) Hernández-Hierro, J. M.; Nogales-Bueno, J.; Rodríguez-Pulido, F. J.; Heredia, F. J. Feasibility study on the use of near-infrared hyperspectral imaging for the screening of anthocyanins in intact grapes during ripening. J. Agric. Food Chem. 2013, 61, 9804-9809.

(21) Nogales-Bueno, J.; Hernández-Hierro, J. M.; Rodríguez-Pulido, F. J.; Heredia, F. J. Determination of technological maturity of grapes and total phenolic compounds of grape skins in red and white cultivars during ripening by near infrared hyperspectral image: A preliminary approach. Food Chem. 2014, 152, 586-591.

(22) Nogales-Bueno, J.; Baca-Bocanegra, B.; Rodríguez-Pulido, F. J.; Heredia, F. J.; Hernández-Hierro, J. M. Use of near infrared hyperspectral tools for the screening of extractable polyphenols in red grape skins. Food Chem. 2015, 172, 559-564.

(23) España Exportación e Inversiones (ICEX). Vinos de España; www.winesfromspain.com/ (accessed May 2014). 
(24) Gordillo, B.; Rodríguez-Pulido, F. J.; Mateus, N.; EscuderoGilete, M. L.; González-Miret, M. L.; Heredia, F. J.; de Freitas, V. Application of LC-MS and tristimulus colorimetry to assess the ageing aptitude of Syrah wine in the Condado de Huelva D.O. (Spain), a typical warm climate region. Anal. Chim. Acta 2012, 732, $162-171$.

(25) Singleton, V. L.; Rossi, J. A., Jr. Colorimetry of total phenolics with phosphomolybdic-phosphotungstic acid reagents. Am. J. Enol. Vitic. 1965, 16, 144-158.

(26) International Organisation of Vine and Wine (OIV). Recuil de Methods Internationals d'Analyse des Vins; OIV: Paris, France, 1990.

(27) Lebart, L.; Morineau, A.; Fénelon, J. P. Tratamiento Estadistico de Datos: Métodos y Programas; Marcombo S.A.: Barcelona, Spain, 1985.

(28) Siesler, H. W.; Ozaky, Y.; Kawata, S.; Heise, H. M. Near Infrared Spectroscopy: Principles, Instruments, Applications; Wiley-VCH: Weinheim, Germany, 2002. 